\title{
Neutrophilic asthma: welcome back!
}

\author{
Peter G. Gibson $\mathbb{1}^{1,2}$ and Paul S. Foster ${ }^{2}$
}

Affiliations: ${ }^{1}$ Dept of Respiratory and Sleep Medicine, John Hunter Hospital, New Lambton Heights, Australia. ${ }^{2}$ Priority Research Centre for Healthy Lungs, The University of Newcastle, Callaghan, Australia.

Correspondence: Peter G. Gibson, University of Newcastle Hunter Medical Research Institute, Dept of Respiratory and Sleep Medicine, Lookout Road, New Lambton Heights, New South Wales, 2305, Australia. E-mail: gibsonahealth.nsw.gov.au

@ERSpublications

Bullone and co-workers show that neutrophilic asthma is a subtype in which the IL-17 pathway seems to be operational. It can be recognised by quantitative immunostaining of bronchial biopsy specimens and may be amenable to specific targeted therapies. http://bit.ly/2llj6Fr

Cite this article as: Gibson PG, Foster PS. Neutrophilic asthma: welcome back!. Eur Respir J 2019; 54: 1901846 [https://doi.org/10.1183/13993003.01846-2019].

What is the immunopathology of neutrophilic asthma, and how can it be recognised? These are important questions, because the answers may pave the way for targeted treatment in this key asthma subtype. Characterising the immunopathology of T2 eosinophilic asthma has led to important therapeutic advances with a range of monoclonal antibodies (mAbs) available for therapy. The close association between sputum and blood eosinophils allows easy recognition of the T2-eosinophilic subtype. But what about the rest? We now know that non-eosinophilic asthma is heterogeneous [1] and so we need to characterise in detail the molecular and cellular nature of these non-T2 subtypes in order to progress development of new therapeutics.

Things are not so simple with neutrophilic asthma. Often its very existence as a subtype is questioned [2]: individuals believe it is COPD and not asthma, dismiss it as a marker of tobacco smoking or corticosteroid exposure (since corticosteroids promote neutrophil survival) [3], suggest it is an artefact of sputum sampling, or believe that it is not relevant in asthma pathogenesis [4]. To further compound the situation, the limited number of treatment trials targeting regulatory aspects of neutrophilia in asthma have been negative $[5,6]$.

Against this background, BulLone et al. [7] sought to characterise the immunopathology of neutrophilic asthma and identify potential clinical markers for this subtype. To gain insights, bronchial biopsies were obtained to sample the lamina propria of the airway at several different levels (lobar, segmental and subsegmental) in 70 patients with a range of asthma severity from mild to severe disease. Subsequent analysis identified a "high neutrophil" subtype, where there was a significant airway wall neutrophilia throughout the bronchial tree. The prevalence of this subtype was 30\%, which is consistent with the prevalence of neutrophilic asthma identified using induced sputum [1]. They then used a range of immunostains to characterise the immunopathology of neutrophilic asthma. The most consistent finding was evidence for presence of the interleukin (IL)-17 pathway. Neutrophil-high patients had a 3-fold increase in cells staining positive for IL-17F+ (also known as the innate T2 cytokine IL-25), which correlated with airway neutrophil numbers. Once smokers were excluded, an increase in IL-17A+ and IL-22+ cells was also revealed. The findings are significant because there were important clinical associations between IL-17F+ cells and exacerbations, increased frequency of oral corticosteroid use, as well as incompletely reversible airflow obstruction. Neutrophilic asthma has previously been shown to be associated with incompletely reversible airflow obstruction [8], which further validates the clinical significance of the findings. 
The IL-17 pathway is linked to the regulation of neutrophils during infections of the lung; however, it is also associated with severe asthma [9-11]. Furthermore, in experimental models, this pathway regulates key pathogenic features of disease, which are often resistant to steroid therapy $[9,11]$. During infections, IL-23 provides a critical signal for the out-growth of CD4 T-helper 17 (Th17) cells, which may also produce IL-22. The study by BulLone et al. [7] now demonstrates in defined neutrophilic asthma cohorts that the expression of IL-23, IL-17A, IL-17F, and IL-22 are increased.

Significantly, this helps identify the neutrophilic asthma subgroup and suggests that this axis plays a pathogenic role. However which cells (only T-cells?) are expressing these cytokines? Are they co-expressed within a given cell? What factors are responsible for driving both the accumulation of neutrophils and eosinophils? For example, Th17 cells have been shown to regulate eosinophil infiltrates in non-Th2 neutrophildominated diseases, such as ulcerative colitis, by granulocyte macrophage colony stimulating factor [12]. Furthermore, in asthma, Th17 cells may also take on a mixed Th2/Th17 phenotype driving both the neutrophilic and T2 arms of the inflammatory cascade $[9,11]$. In addition, Th17 cells do not always express IL-22 and there is also a distinct lineage of IL-22 producing T-helper cells (that do not express IL-17) [13].

Interestingly, the neutrophilic patients also had eosinophils present in the lamina propria, elevated serum $\operatorname{IgE}$, and most were atopic. The high prevalence of atopy parallels what is seen in neutrophilic asthma identified by induced sputum [1]. This raises the question as to the origin of airway disease and relevant pathogenic pathways. Are these individuals initially T2 and then over time through exposure to specific infections and allergens become a dominant neutrophilic phenotype with pathways that can drive neutrophilia and eosinophilia? Or are these individuals a distinct Th17 endotype? IgE can activate B-cells, which results in increased cytokine production from Th2 cells through an antigen-specific process and this mechanism of T-helper cell activation may operate for Th17 cells in neutrophilic asthma. In children with asthma, neutrophilic asthma is a very uncommon subtype, whereas it emerges in adults with asthma [14]. These findings suggest that neutrophilic asthma may evolve with life exposure, and if so, it may also regress, possibly with therapy. Further characterisation of the types of T-helper cells and cytokine levels in these NA patients will be invaluable. As such, the observations of BuLLone et al. [7] are very interesting and defining.

What does this mean for targeted therapy of neutrophilic asthma? Brodalumab is a human anti-IL-17 receptor $\mathrm{A} \mathrm{mAb}$ that inhibits IL-17A and IL-17F [15]. It is effective in psoriasis, but a trial in asthma was negative [5]. This trial did not select patients for presence of the IL-17 pathway. This is reminiscent of the early clinical use of anti-IL-5 mAbs, where selecting patients with an active eosinophilic pathway was crucial to demonstrating efficacy. Is finding a treatment for neutrophilic asthma a case of "déjà $v u$, all over again"? The work from Bullone et al. [7] shows that not all asthmatics exhibit an IL-17 phenotype, and shows us that bronchial biopsy can be used to recognise this pathway in asthma. It is now a simple step to apply inhibition of IL-17 to a subgroup of asthma patients showing activity of this pathway.

Given the IgE results and the co-identification of eosinophils and neutrophils in the lamina propria of patients with neutrophilic asthma, is there a place for T2 (mAb) therapy in these patients? This is a really interesting possibility. It would be possible to assess changes in the IL-17 pathway using bronchial biopsy in patients treated with $\mathrm{T} 2 \mathrm{mAbs}$ to gain some insight into this question. At least $50 \%$ of the neutrophil-high patients had blood eosinophils of $3 \%$ or greater [7], meaning they could qualify for T2 mAb therapy.

Neutrophilic asthma is an asthma subtype where the IL-17 pathway seems to be operational. It can be recognised by quantitative immunostaining of bronchial biopsy specimens, and may be amenable to specific targeted therapies. Testing these therapies on unselected patients may not identify their clinical efficiency because of the low prevalence of neutrophilic asthma, and risks premature discontinuation of the development of new asthma drugs. There are now clear directions for research to characterise the mechanisms of neutrophilic asthma, to develop simpler ways to identify IL-17 asthma, and to test therapies for this pathway in patients who have IL-17 driven asthma.

Conflict of interest: P.G. Gibson reports grants and personal fees from AstraZeneca, GlaxoSmithKline, Novartis and Sanofi, outside the submitted work. P.S. Foster has nothing to disclose.

Support statement: The preparation of this article was supported by National Health and Medical Research Council Practitioner Fellowship APP1155810. The funding source had no role in the writing of the report or decision to submit the article for publication. Funding information for this article has been deposited with the Crossref Funder Registry.

\section{References}

1 Simpson JL, Scott R, Boyle MJ, et al. Inflammatory subtypes in asthma: assessment and identification using induced sputum. Respirology 2006; 11: 54-61. 
2 Sze E, Bhalla A, Nair P. Mechanisms and therapeutic strategies for non-T2 asthma. Allergy 2019; in press [https:// doi.org/10.1111/all.13985]

3 Cowan DC, Cowan JO, Palmay R, et al. Effects of steroid therapy on inflammatory cell subtypes in asthma. Thorax 2010; 65: 384-390.

4 Snelgrove RJ, Patel DF, Patel T, et al. The enigmatic role of the neutrophil in asthma: Friend, foe or indifferent? Clin Exp Allergy 2018; 48: 1275-1285.

5 Busse WW, Holgate S, Kerwin E, et al. Randomized, double-blind, placebo-controlled study of brodalumab, a human anti-IL-17 receptor monoclonal antibody, in moderate to severe asthma. Am J Respir Crit Care Med 2013; 188: 1294-1302.

6 O'Byrne PM, Metev H, Puu M, et al. Efficacy and safety of a CXCR2 antagonist, AZD5069, in patients with uncontrolled persistent asthma: a randomised, double-blind, placebo-controlled trial. Lancet Respir Med 2016; 4 : 797-806.

7 Bullone M, Carriero V, Bertolini F, et al. Elevated serum IgE, oral corticosteroid dependence and IL-17/22 expression in highly neutrophilic asthma. Eur Respir J 2019; 54: 1900068.

8 Shaw DE, Berry MA, Hargadon B, et al. Association between neutrophilic airway inflammation and airflow limitation in adults with asthma. Chest 2007; 132: 1871-1875.

9 Bartemes KR, Iijima K, Kobayashi T, et al. IL-33-responsive lineage- CD25+ CD44(hi) lymphoid cells mediate innate type 2 immunity and allergic inflammation in the lungs. J Immunol 2012; 188: 1503-1513.

10 Chesne J, Braza F, Mahay G, et al. IL-17 in severe asthma. Where do we stand? Am J Respir Crit Care Med 2014; 190: 1094-1101.

11 Halim TY, Steer CA, Matha L, et al. Group 2 innate lymphoid cells are critical for the initiation of adaptive T helper 2 cell-mediated allergic lung inflammation. Immunity 2014; 40: 425-435.

12 Keely S, Foster PS. Stop press: eosinophils drafted to join the Th17 team. Immunity 2015; 43: 7-9.

13 Plank MW, Kaiko GE, Maltby S, et al. Th22 cells form a distinct Th lineage from Th17 cells in vitro with unique transcriptional properties and Tbet-dependent Th1 plasticity. J Immunol 2017; 198: 2182-2190.

14 Wang F, He XY, Baines KJ, et al. Different inflammatory phenotypes in adults and children with acute asthma. Eur Respir J 2011; 38: 567-574.

15 Sakkas LI, Zafiriou E, Bogdanos DP. Mini review: new treatments in psoriatic arthritis. focus on the IL-23/17 axis. Front Pharmacol 2019; 10: 872. 